\title{
The L3MBTL3 methyl-lysine reader domain functions as a dimer
}

\author{
Brandi M. Baughman ${ }^{a}$, Samantha G. Pattenden ${ }^{a}$, Jacqueline L. Norris ${ }^{a}$, Lindsey I. James ${ }^{a}$, \\ and Stephen V. Frye ${ }^{*}$,a \\ ${ }^{a}$ Center for Integrative Chemical Biology and Drug Discovery, Division of Chemical Biology and \\ Medicinal Chemistry, UNC Eshelman School of Pharmacy, University of North Carolina at Chapel \\ Hill, Chapel Hill, North Carolina 27599, USA
}

\begin{abstract}
L3MBTL3 recognizes mono- and dimethylated lysine residues on histone tails. The recently reported X-ray co-crystal structures of the chemical probe UNC1215 and inhibitor UNC2533 bound to the methyl-lysine reading MBT domains of L3MBTL3 demonstrate a unique and flexible 2:2 dimer mode of recognition. In this study, we describe our in vitro analysis of L3MBTL3 dimerization via its MBT domains and additionally show that this dimerization occurs within a cellular context in the absence of small molecule ligands. Furthermore, mutations to the first and second MBT domains abrogated L3MBTL3 dimerization both in vitro and in cells. These observations are consistent with the hypothesis that L3MBTL3 engages methylated histone tails as a dimer while carrying out its normal function and provides an explanation for the presence of repeated MBT domains within L3MBTL3.
\end{abstract}

Eukaryotic DNA is packaged in the nucleus by histone proteins to form nucleosomes, the repeating subunit of chromatin ((1-3)). Precise regulation of chromatin structure is essential for normal cellular function and is guided in part by post-translational modifications (PTMs) of histones. The unstructured tails of histones are rich in PTMs including methylation, acetylation, phosphorylation, and ubiquitination $((4,5))$. Histone PTMs can directly alter the physical structure of chromatin ((6)) and function in the context of a 'histone code' to coordinate the binding of effector proteins that elicit selective changes in gene expression and other chromatintemplated biological processes like DNA repair ((7-9)). Of these histone PTMs, methylation at lysine residues has been shown to play a dynamic role in controlling access of DNA transcriptional machinery, protein expression, and cell function ((10-14)), all of which are dependent upon the location and degree of methylation ((15)). The functional consequences of lysine methylation largely rely upon the recruitment of chromatin regulatory proteins that contain, or are in complex with, a methyl-lysine (Kme) 'reader' ((7)).

Recently, interest in small molecule antagonists of methyl-lysine readers has emerged ((1619)), spurred in part by the rapid clinical translation of small molecule antagonists of bromodomains, the readers of acetylated lysine ((20)). Previous work in our group has

*svfrye@email.unc.edu; Fax: +1 919-843-8465; Tel: +1 919-843-5486.

Supporting Information Available: This information is available free of charge via the Internet. 
focused on the reading of Kme marks by malignant brain tumor (MBT) domain-containing proteins. MBT domains selectively recognize mono- and dimethyl-lysine residues utilizing an aromatic cage that is sterically optimized for these methylation states $((21))$ and a required aspartic acid residue that hydrogen bonds to the protonated side-chain amine ( $(22$, 23)). Interestingly, all MBT proteins contain two to four repeats of the eponymous domain and there is some genetic evidence of function for these multiple binding sites $((24))$, although prior crystallography has demonstrated engagement of only a single domain in binding Kme containing peptides ((23)). In contrast to some other Kme readers, MBT domains show minimal sequence specificity in vitro for mono- and dimethyl-lysine histone peptides, with affinities typically in the $20-50 \mu \mathrm{M}$ range. In light of the provocative tri-fold propeller structure of the three MBT domains of the first crystallized member of this family ((24)), L3MBTL1, that constrains all copies of the domain to face in a common direction as if arranged to engage its substrate, we were curious as to how these proteins function in vivo. Could the repeated domains collaborate in some fashion to create a specific and high-affinity interaction for chromatin? Seminal studies of the function of L3MBTL1 have proposed models that invoke monomeric or dimeric versions of the protein potentially reading multiple histone modifications to elicit the specific regulation of facultative heterochromatin $((25))$, but a molecular level understanding of these models remains elusive.

Similar to L3MBTL1, the closely related protein, L3MBTL3, also contains three MBT repeats (Figure 1A) and has been shown to play a role in meduloblastoma formation ((16)) and normal hematopoiesis in humans $((17,22,25-28))$. Our group discovered a potent and selective L3MBTL3 chemical probe, UNC1215 (Figure 1C) ((28)), and has recently reported structure-activity relationships for classes of compounds related to UNC1215 ((29, 30)). The X-ray co-crystal structures of both UNC1215 (pdb 4FL6) and a structurally distinct ligand, UNC2533 (pdb 4L59), show that these compounds bind L3MBTL3 as a 2:2 dimer with protein (Figure 1 and Figure S1). The small molecule ligands bridge two of the three MBT repeats (one from each protein unit) in a face to face arrangement wherein two copies of protein sandwich two ligand molecules (Figure 1B). Specifically, it is thought that ligand binding is largely driven by interaction with the Kme binding pocket in domain 2 where the inhibitor makes a key hydrogen bond with residue D381, and that binding is further enhanced by engaging the first domain of the second protein in the L3MBTL3 dimer. Mutation of the first MBT domain (D274A) weakens ligand binding via disruption of L3MBTL3 dimerization.

Size exclusion chromatography utilizing the MBT repeats of L3MBTL3 (as opposed to the full length protein) also indicated that in the absence of UNC1215 there exists a small population of dimer that is stabilized and amplified upon addition of compound (28). These findings suggested that the mechanism of ligand recognition observed in the crystal lattice might also be operative in solution and led us to hypothesize that L3MBTL3 exists and functions as a dimer in vivo as well. In contrast, L3MBTL1 has so far only been shown to interact with small molecule ligands as a monomer and shows no evidence of dimerization via its MBT repeats $((25,31))$. To date there has been little reported on the mechanism of action of endogenous L3MBTL3; thus, elucidating its binding mode within a cellular context could provide further insight into Kme recognition by the MBT family of chromatin 
regulators. Herein we quantitate the in vitro dimerization of L3MBTL3 in the absence and presence of UNC1215 and report the identification of L3MBTL3 dimerization in vivo that depends upon Kme recognition.

\section{Results and Discussion}

To validate and quantitate L3MBTL3 dimerization in vitro, we designed an AlphaScreen assay to assess dimer populations in the presence and absence of UNC1215 (Figure 2).

Using biotinylated and 6His-tagged versions of the three MBT domains of L3MBTL3 with streptavidin-conjugate donor and nickel-chelate acceptor beads, we were able to observe L3MBTL3 dimerization with an affinity of $40.6 \mu \mathrm{M}$ in the absence of ligand (Figure 2, Table 1). This dimerization affinity increased more than 10 -fold to $1.1 \mu \mathrm{M}(\mathrm{p}<0.0001)$ in the presence of UNC1215, thus supporting the enhanced dimerization of L3MBTL3 upon ligand binding. It should be noted that the affinity of UNC1215 for L3MBTL3 as measured by ITC is $120 \mathrm{nM}$, and this potent binding was previously shown to be dependent upon L3MBTL3 dimerization via site directed mutagenesis ((28)). To explore the effect of a candidate in vivo substrate on L3MBTL3 dimerization, unlabeled H4K20me2 peptide was similarly added to a mixture of biotinylated L3MBTL3 and 6His-L3MBTL3. Enhanced L3MBTL3 dimerization was observed (Table 1 and Figure S2), and although the affinity change was not as dramatic as that caused by addition of UNC1215, it was still significant $(14.1 \mu \mathrm{M}$, $\mathrm{p}<0.0001)$. This was anticipated due to the fact that $\mathrm{H} 4 \mathrm{~K} 20 \mathrm{me} 2$ is a much weaker ligand as measured by ITC (Figure S4). As expected, the dimer population was not enhanced in the presence of a negative control compound, UNC1079, which is structurally similar to UNC1215 but a significantly less potent ligand (Figure 1C, Table 1) ((28)).

To determine the dependence of L3MBTL3 dimerization on the function of individual MBT domains, we compared the degree of dimerization of wildtype biotinylated L3MBTL3 to 6His-tagged L3MBTL3 MBT domain 1 and 2 mutants, D274A and D381A respectively, in the presence and absence of UNC1215 and UNC1079 (Table 1 and Figure S3). Based on prior ITC results with these mutants as well as X-ray co-crystal studies ((28)), we anticipated that these mutants would abrogate dimerization if it is induced by ligand binding to both MBT pockets containing these residues. In the absence of ligand, weak dimerization was observed between each mutant and wildtype L3MBTL3, with affinities for each greater than $100 \mu \mathrm{M}$. This is reflective the small dimer population typically observed in the absence of ligand. When incubated with UNC1215, no AlphaScreen signal was observed with either domain 1 or domain 2 mutant, indicative of no dimerization (Table 1 and Figure S3). This is because in the presence of mutated 6His-L3MBTL3, UNC1215 is bound exclusively by the biotinylated wildtype L3MBTL3 homodimer protein population. The equilibrium in Figure $2 \mathrm{~A}$ is therefore shifted entirely towards homodimerization of wildtype biotin-L3MBTL3 and results in a decreased assay signal (Figure S3). In contrast, incubation with UNC1079 does not result in a similar equilibrium shift and the weak binding curves observed closely mimic those without ligand. To assure that the observed dimerization of L3MBTL3 proteins was not attributed to aggregation or non-specific binding, we measured the interaction between biotinylated wildtype L3MBTL3 and 6His-CBX7, a chromodomain-containing Kme reading 
protein also studied in our lab. No protein-protein interactions were observed between L3MBTL3 and CBX7 (Table 1 and Figure S3).

To further confirm our results and validate that the dimerization of L3MBTL3 is a specific interaction, we repeated the above experiments in a LANCE assay format ((30)). LANCE is a TR-FRET (time-resolved fluorescence energy transfer) assay, whereby the distance between protein partners required for a robust signal $(\sim 10 \mathrm{~nm})$ is much shorter than that required for the AlphaScreen assay ( $200 \mathrm{~nm}$ ). The LANCE results correlated very well with the AlphaScreen data, further confirming that ligand binding enhances dimerization of L3MBTL3 and that both MBT domains 1 and 2 are involved in substrate recognition (Tables 1 and S1, and Figure S2).

Collectively, these results show that L3MBTL3 dimerizes via its MBT domains in vitro and that this dimer population, while amplified in the presence of ligand, also exists in the absence of ligand. These data clearly support our conjecture that L3MBTL3 utilizes at least two of its three MBT repeats during substrate recognition in a fashion similar to our prior crystallography results ((28)). Unfortunately we are unable to readily explore the functional consequences of mutating the third MBT repeat of L3MBTL3 as mutation of the key residue in this domain resulted in protein that had limited solubility and was problematic to purify.

While the biological function of L3MBTL3 in vivo is still largely unknown, studies have indicated that MBT domains assist in targeting chromatin regulatory complexes to appropriate genomic loci via mono- or dimethyl-lysine recognition within histone tails ((23, $25,32-34)$ ). The molecular details of this recognition including the identification of the endogenous ligand(s) and the role of multiple MBT repeats remain unexplored. Based on our structural data with small molecule ligands and the evidence presented above, we sought to investigate whether L3MBTL3 engages its cognate targets via dimerization of its MBT domains within a cellular context.

In addition to the MBT domains, L3MBTL3 has a zinc finger, a putative RNA-binding domain, and a C-terminal sterile alpha motif (SAM) domain. SAM domains are proteinprotein interaction domains that can homo- or heterodimerize and often play a role in the formation of cellular protein complexes ((35)). Because it has been proposed that the SAM domain of L3MBTL3 is capable of polymer formation ((36)), we decided to examine the ability of L3MBTL3 to dimerize in cells with and without the SAM domain via coimmunoprecipitation (co-IP) experiments.

To assess L3MBTL3 dimerization, a stable cell line expressing N-terminal GFP-tagged full length L3MBTL3 (GFP-L3; Figure 3A) in HeLa cells was prepared. HeLa cells were chosen because they have very low expression levels of endogenous L3MBTL3, which was necessary in order to study the dimerization between two introduced fusion proteins by coIP ((30)). Western blot analysis of GFP-L3 cells transfected with a FLAG-tagged fusion protein of the 3 MBT domains of L3MBTL3 lacking the SAM domain (3MBT-L3; Figure 3A) confirmed cellular dimerization (Figure 3B, lane 1), possibly via binding of the MBT domains to an endogenous methyl-lysine substrate. Upon treatment with UNC1215, dimerization is observed to a similar extent (Figure 3B, lane 2). Consistent with this 
conjecture, dimerization was disrupted when GFP-L3 cells were transfected with the FLAG-3MBT mutants, D274A (3MBT-D274A; Figure 3A and 3B, lane 3) and D381A (3MBT-D381A; Figure 3A and 3B, lane 5), as these mutants decrease or abrogate ligand binding respectively by preventing dimerization. This is further supported by the fact that L3MBTL3 dimerization was not observed with either mutant in the presence of the potent ligand, UNC1215 (Figure 3B, lanes 4 and 6). These data confirm that functional domain 1 and $2 \mathrm{Kme}$ binding pockets are required for dimerization when the SAM domain is missing in one partner.

Next, GFP-L3 cells were transfected with a FLAG fusion protein of full length wild type L3MBTL3 (FullL-L3; Figure 3A). Similarly, dimerization was observed between the full length GFP- and FLAG-tagged L3MBTL3 proteins in both the absence and presence of UNC1215 (Figure 3B, lanes 7 and 8). Introduction of the corresponding full length D274A and D381A mutants (Figure 3A, FullL-D274A and FullL-D381A) produced similar results to those observed with the mutated MBT only constructs (Figure 3B, lanes 9-12). Overall, mutation of the key Kme reading domains in the context of full length L3MBTL3 disrupts its dimerization. It should be noted that the co-IPs with FullL-D274A produced variable results, and while the dominant observation is depicted in Figure 3B, in some cases dimerization was observed (Figure S6). Inconsistency in dimerization of this construct may indicate that other domains, such as the SAM domain, contribute to a weakly stable dimer population and is consistent with a dominant role of the still functional, D381 containing pocket in this mutant. The addition of UNC1215 in each case did not affect the co-IP readout of dimerization, indicating that the Kme pocket functional dependence of dimerization in L3MBTL3 is an endogenous event that phenocopies the effect of chemical probe binding.

In conclusion, we have shown that L3MBTL3 exists and functions as a dimer both in vitro and in cells. Dimerization via the MBT domains is observed in both AlphaScreen and LANCE assays in vitro and is amplified in the presence of UNC1215 and H4K20me2. Our co-IP results support that dimerization within a cellular context is also due to interactions between the MBT domains and their ligands and requires participation of both the first and second MBT domains within each protein. Dimerization via the MBT domains may serve to explain how L3MBTL3 engages PTMs on histone tails and suggests a role for the repeating MBT domains within L3MBTL3 and possibly other Kme readers in this family. Furthermore, MBT-containing proteins have been shown to play a role in chromatin compaction and this novel binding mode via dimerization may provide insight into the mechanism utilized ((25)).

\section{Methods}

Details of experimental procedures are outlined in the Supporting Information.

\section{Supplementary Material}

Refer to Web version on PubMed Central for supplementary material. 


\section{Acknowledgements}

We thank the Structural Genomics Consortium for providing the constructs and/or plasmids for L3MBTL3 and CBX7. This research was supported by the National Institute of General Medical Sciences, US National Institutes of Health (NIH, grant R01GM100919), the Carolina Partnership, and the University Cancer Research Fund.

\section{References}

1. Kornberg RD. Chromatin structure: a repeating unit of histones and DNA. Science. 1974; 184:868871. [PubMed: 4825889]

2. Van Holde KE, Allen JR, Tatchell K, Weischet WO, Lohr D. DNA-histone interactions in nucleosomes. Biophys J. 1980; 32:271-282. [PubMed: 6788105]

3. Luger K, Mader AW, Richmond RK, Sargent DF, Richmond TJ. Crystal structure of the nucleosome core particle at 2.8 A resolution. Nature. 1997; 389:251-260. [PubMed: 9305837]

4. Kouzarides T. Chromatin modifications and their function. Cell. 2007; 128:693-705. [PubMed: 17320507]

5. Tan M, Luo H, Lee S, Jin F, Yang JS, Montellier E, Buchou T, Cheng Z, Rousseaux S, Rajagopal N, Lu Z, Ye Z, Zhu Q, Wysocka J, Ye Y, Khochbin S, Ren B, Zhao Y. Identification of 67 histone marks and histone lysine crotonylation as a new type of histone modification. Cell. 2011; 146:10161028. [PubMed: 21925322]

6. Shogren-Knaak M, Ishii H, Sun JM, Pazin MJ, Davie JR, Peterson CL. Histone H4-K16 acetylation controls chromatin structure and protein interactions. Science. 2006; 311:844-847. [PubMed: 16469925]

7. Taverna SD, Li H, Ruthenburg AJ, Allis CD, Patel DJ. How chromatin-binding modules interpret histone modifications: lessons from professional pocket pickers. Nat Struct Mol Biol. 2007; 14:1025-1040. [PubMed: 17984965]

8. Strahl BD, Allis CD. The language of covalent histone modifications. Nature. 2000; 403:41-45. [PubMed: 10638745]

9. Jenuwein T, Allis CD. Translating the histone code. Science. 2001; 293:1074-1080. [PubMed: 11498575]

10. Bernstein BE, Humphrey EL, Erlich RL, Schneider R, Bouman P, Liu JS, Kouzarides T, Schreiber SL. Methylation of histone H3 Lys 4 in coding regions of active genes. Proc Natl Acad Sci U S A. 2002; 99:8695-8700. [PubMed: 12060701]

11. Santos-Rosa H, Schneider R, Bannister AJ, Sherriff J, Bernstein BE, Emre NC, Schreiber SL, Mellor J, Kouzarides T. Active genes are tri-methylated at K4 of histone H3. Nature. 2002; 419:407-411. [PubMed: 12353038]

12. Schotta G, Ebert A, Krauss V, Fischer A, Hoffmann J, Rea S, Jenuwein T, Dorn R, Reuter G. Central role of Drosophila SU(VAR)3-9 in histone H3-K9 methylation and heterochromatic gene silencing. EMBO J. 2002; 21:1121-1131. [PubMed: 11867540]

13. Stewart MD, Li J, Wong J. Relationship between histone H3 lysine 9 methylation, transcription repression, and heterochromatin protein 1 recruitment. Mol Cell Biol. 2005; 25:2525-2538. [PubMed: 15767660]

14. Schwartz YB, Pirrotta V. Polycomb silencing mechanisms and the management of genomic programmes. Nat Rev Genet. 2007; 8:9-22. [PubMed: 17173055]

15. Zhang Y, Reinberg D. Transcription regulation by histone methylation: interplay between different covalent modifications of the core histone tails. Genes Dev. 2001; 15:2343-2360. [PubMed: 11562345]

16. Herold JM, Ingerman LA, Gao C, Frye SV. Drug discovery toward antagonists of methyl-lysine binding proteins. Curr Chem Genom. 2011; 5:51-61.

17. James LI, Frye SV. Targeting chromatin readers. Clin Pharmacol Ther. 2013; 93:312-314. [PubMed: 23403847]

18. Wagner EK, Nath N, Flemming R, Feltenberger JB, Denu JM. Identification and Characterization of Small Molecule Inhibitors of a Plant Homeodomain Finger. Biochemistry. 2012; 51:8293-8306. [PubMed: 22994852] 
19. Simhadri C, Daze KD, Douglas SF, Quon TTH, Dev A, Gignac MC, Peng F, Heller M, Boulanger MJ, Wulff JE, Hof F. Chromodomain antagonists that target the polycomb-group methyllysine reader protein Chromobox homolog 7 (CBX7). J Med Chem. 2014; 57:2874-2883. [PubMed: 24625057]

20. Brennan P, Filippakopoulos P, Knapp S. The therapeutic potential of acetyl-lysine and methyllysine effector domains. Drug Discov Today Ther Strateg. 2012; 9:e101-e110.

21. Gao C, Herold JM, Kireev D, Wigle T, Norris JL, Frye S. Biophysical Probes Reveal a "Compromise" Nature of the Methyl-lysine Binding Pocket in L3MBTL1. J Am Chem Soc. 2011; 133:5357-5362. [PubMed: 21428286]

22. Bonasio R, Lecona E, Reinberg D. MBT domain proteins in development and disease. Semin Cell Dev Biol. 2010; 21:221-230. [PubMed: 19778625]

23. Nady N, Krichevsky L, Zhong N, Duan S, Tempel W, Amaya MF, Ravichandran M, Arrowsmith $\mathrm{CH}$. Histone recognition by human malignant brain tumor domains. J Mol Biol. 2012; 423:702718. [PubMed: 22954662]

24. Wang WK, Tereshko V, Boccuni P, MacGrogan D, Nimer SD, Patel DJ. Malignant Brain Tumor Repeats: A Three-Leaved Propeller Architecture with Ligand/Peptide Binding Pockets. Structure. 2003; 11:775-789. [PubMed: 12842041]

25. Trojer P, Li G, Sims RJ 3rd, Vaquero A, Kalakonda N, Boccuni P, Lee D, Erdjument-Bromage H, Tempst P, Nimer SD, Wang YH, Reinberg D. L3MBTL1, a histone-methylation-dependent chromatin lock. Cell. 2007; 129:915-928. [PubMed: 17540172]

26. Botuyan MV, Lee J, Ward IM, Kim J-E, Thompson JR, Chen J, Mer G. Structural Basis for the Methylation State-Specific Recognition of Histone H4-K20 by 53BP1 and Crb2 in DNA Repair. Cell. 2006; 127:1361-1373. [PubMed: 17190600]

27. Wigle TJ, Herold JM, Senisterra GA, Vedadi M, Kireev DB, Arrowsmith CH, Frye SV, Janzen WP. Screening for Inhibitors of Low-Affinity Epigenetic Peptide-Protein Interactions: An AlphaScreenTM-Based Assay for Antagonists of Methyl-Lysine Binding Proteins. J Biomol Screen. 2010; 15:62-71. [PubMed: 20008125]

28. James LI, Barsyte-Lovejoy D, Zhong N, Krichevsky L, Korboukh VK, Herold JM, MacNevin CJ, Norris JL, Sagum CA, Tempel W, Marcon E, Guo H, Gao C, Huang X-P, Duan S, Emili A, Greenblatt JF, Kireev DB, Jin J, Janzen WP, Brown PJ, Bedford MT, Arrowsmith CH, Frye SV. Discovery of a chemical probe for the L3MBTL3 methyllysine reader domain. Nat Chem Biol. 2013; 9:184-191. [PubMed: 23292653]

29. Camerino MA, Zhong N, Dong A, Dickson BM, James LI, Baughman BM, Norris JL, Kireev DB, Janzen WP, Arrowsmith CH, Frye SV. The structure-activity relationships of L3MBTL3 inhibitors: flexibility of the dimer interface. MedChemComm. 2013; 4:1501-1507. [PubMed: 24466405]

30. James LI, Korboukh VK, Krichevsky L, Baughman BM, Herold JM, Norris JL, Jin J, Kireev DB, Janzen WP, Arrowsmith CH, Frye SV. Small-Molecule Ligands of Methyl-Lysine Binding Proteins: Optimization of Selectivity for L3MBTL3. J Med Chem. 2013; 56:7358-7371. [PubMed: 24040942]

31. Herold JM, Wigle TJ, Norris JL, Lam R, Korboukh VK, Gao C, Ingerman LA, Kireev DB, Senisterra G, Vedadi M, Tripathy A, Brown PJ, Arrowsmith CH, Jin J, Janzen WP, Frye SV. Small-Molecule Ligands of Methyl-Lysine Binding Proteins. J Med Chem. 2011; 54:2504-2511. [PubMed: 21417280]

32. Takada Y, Isono K.-i. Shinga J, Turner JMA, Kitamura H, Ohara O, Watanabe G, Singh PB, Kamijo T, Jenuwein T, Burgoyne PS, Koseki H. Mammalian Polycomb Scmh1 mediates exclusion of Polycomb complexes from the XY body in the pachytene spermatocytes. Development. 2007; 134:579-590. [PubMed: 17215307]

33. Trojer P, Cao AR, Gao Z, Li Y, Zhang J, Xu X, Li G, Losson R, Erdjument-Bromage H, Tempst P, Farnham PJ, Reinberg D. L3MBTL2 Protein Acts in Concert with PcG Protein-Mediated Monoubiquitination of H2A to Establish a Repressive Chromatin Structure. Mol Cell. 2011; 42:438-450. [PubMed: 21596310]

34. Ogawa H, Ishiguro K.-i. Gaubatz S, Livingston DM, Nakatani Y. A Complex with Chromatin Modifiers That Occupies E2F- and Myc-Responsive Genes in G0 Cells. Science. 2002; 296:11321136. [PubMed: 12004135] 
35. Kim CA, Gingery M, Pilpa RM, Bowie JU. The SAM domain of polyhomeotic forms a helical polymer. Nat Struct Biol. 2002; 9:453-457. [PubMed: 11992127]

36. Knight MJ, Leettola C, Gingery M, Li H, Bowie JU. A human sterile alpha motif domain polymerizome. Protein Sci. 2011; 20:1697-1706. [PubMed: 21805519] 

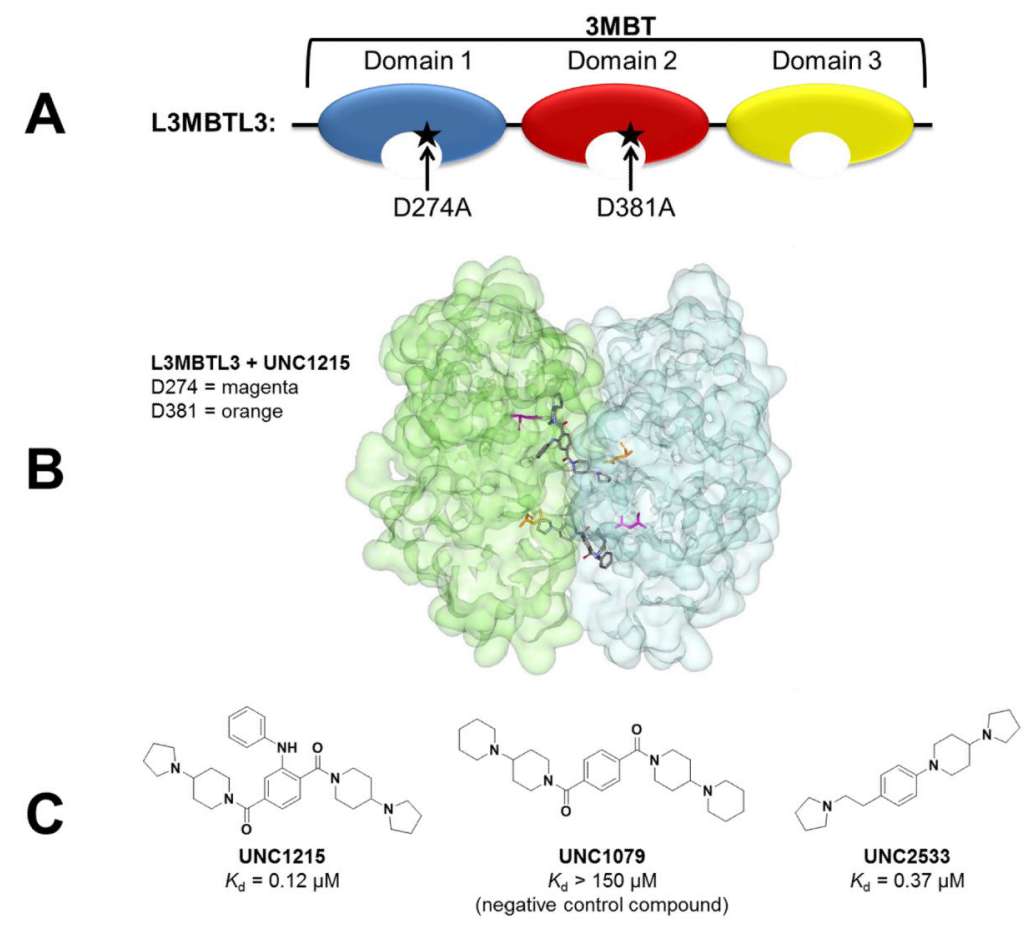

Figure 1.

L3MBTL3 and its small molecule ligands. A) The 3MBT domains of L3MBTL3 were used in biochemical assays to discover the small molecule probe UNC1215. Mutations to domain 1 (D274A) and domain 2 (D381A) decreased or abolished the binding of UNC1215 to L3MBTL3 respectively. B) The x-ray crystal structure of the 2:2 dimer of L3MBTL3 and UNC1215 shows a face to face arrangement wherein two copies of protein (one copy shown in green and one in blue) sandwich two ligand molecules (pdb 4FL6). Residue D274 is highlighted in magenta and residue D381 is highlighted in orange. C) Structures of small molecule inhibitors of L3MBTL3 and their affinities as measured by isothermal titration calorimetry. UNC1079 is a structurally similar but significantly less potent negative control compound. 
A

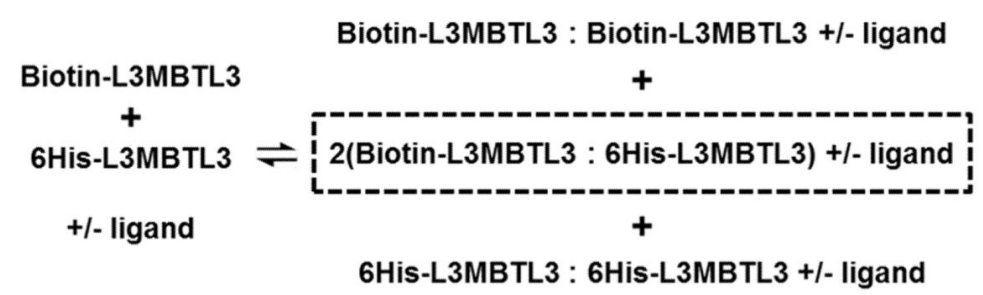

B

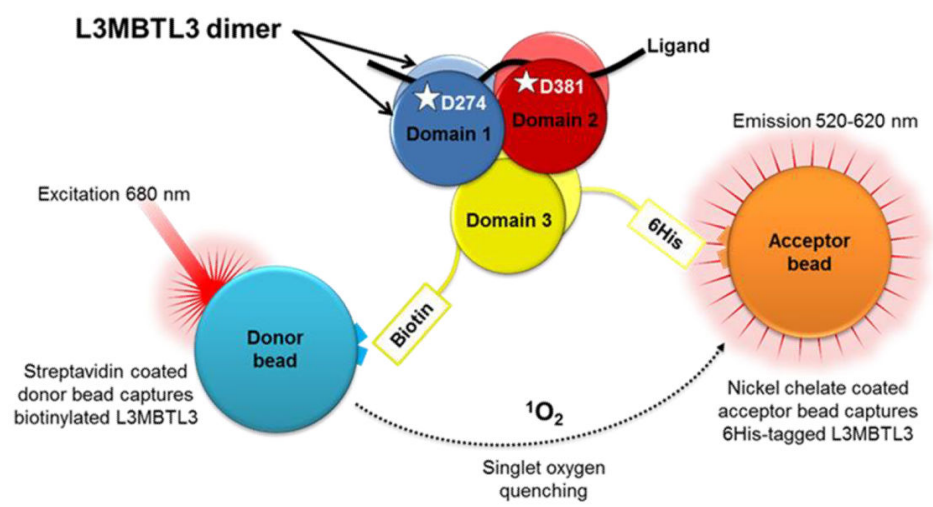

Figure 2.

Schematic of the evaluation of L3MBTL3 dimerization in vitro. A) Equilibrium between monomer and dimer populations of L3MBTL3 in the presence and absence of ligands. Homodimer populations exist for the biotin and 6His labeled protein versions of L3MBTL3; however, only heterodimerization between biotin and 6His proteins result in a measureable assay signal in AlphaScreen and LANCE (outlined in dashed box). Ligands are histone H4 dimethylated on lysine 20 (H4K20me2; residues 12-27), UNC1215, or UNC1079. B) The AlphaScreen assay developed to measure heterodimerization of L3MBTL3 in the presence or absence of ligand. 

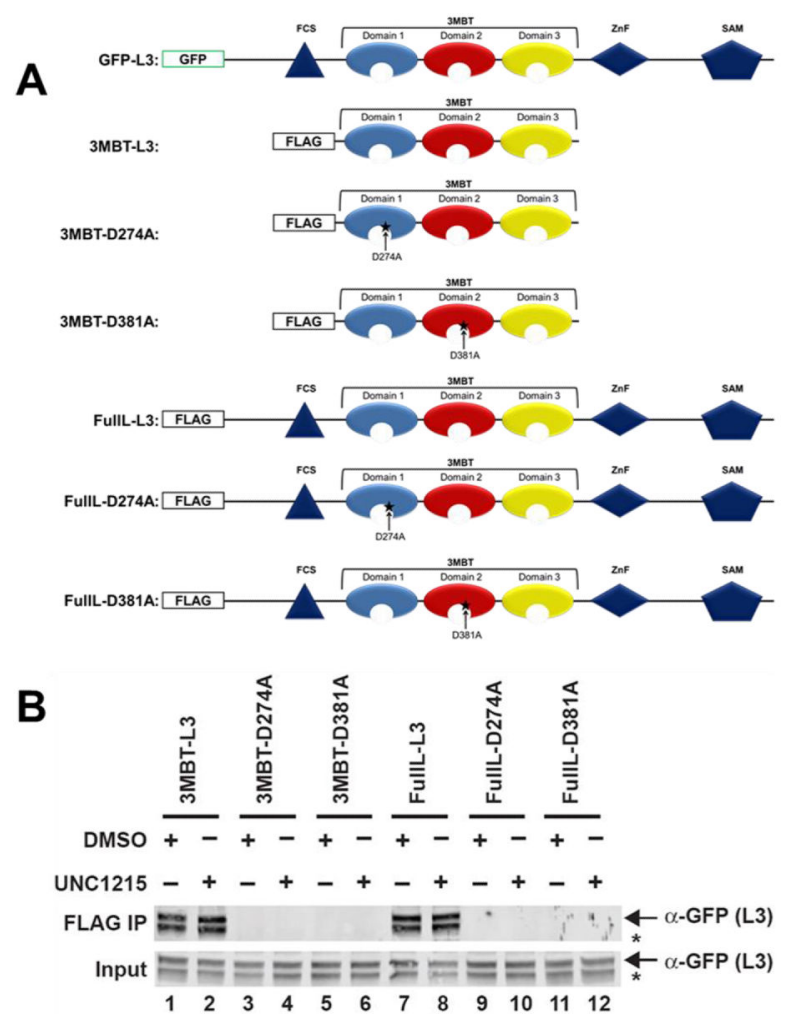

Figure 3.

L3MBTL3 co-IP experiments to evaluate cellular dimerization. A) Schematics of plasmids used for co-IP experiments showing a putative phenylalanine-cysteine-serine nucleic acidbinding domain (FCS), a Zinc finger ( $\mathrm{ZnF}$ ), and a sterile a motif (SAM) domain, in addition to three MBT repeat sequences (blue, red and yellow). Mutations made in the first MBT domain (D274A) and in the second MBT domain (D381A) are represented by stars. B) HeLa cells stably expressing GFP-L3 were transfected with various FLAG-tagged plasmids. Western blot analysis revealed that both 3MBT and full length L3MBTL3 effectively binds GFP-tagged full length L3MBTL3 (IP: FLAG; IB:GFP). The D274A and D381A MBT domain mutations inhibit L3MBTL3 dimerization. The L3MBTL3 band is denoted with an arrow. *Denotes a non-specific band verified to not be L3MBTL3 (Figure S7). 


\section{Table 1}

Summary of L3MBTL3 protein-protein interactions as measured by AlphaScreen. In all instances biotinylated wildtype L3MBTL3 (150 nM) serves as the AlphaScreen or LANCE donor. ${ }^{a}$

\begin{tabular}{|c|c|c|c|}
\hline Donor & Ligand & AlphaScreen $\mathrm{IC}_{50}(\mu \mathrm{M})$ & LANCE IC $\left._{50} \mu \mathrm{M}\right)$ \\
\hline \multirow{4}{*}{ 6His-L3MBTL3 } & DMSO & $40.63 \pm 2.29(6)$ & $32.48 \pm 2.29(6)$ \\
\hline & $\mathrm{H} 4 \mathrm{~K} 20 \mathrm{me} 2$ & $14.11 \pm 2.73(6)$ & $16.43 \pm 1.94(6)$ \\
\hline & UNC1215 & $1.13 \pm 0.11(6)$ & $1.41 \pm 0.30(6)$ \\
\hline & UNC1079 & $41.22 \pm 3.75(3)$ & $30.44 \pm 1.08(3)$ \\
\hline \multirow{3}{*}{ 6His-D381A } & DMSO & $>100(6)$ & $>100(6)$ \\
\hline & UNC1215 & No binding (6) & No binding (6) \\
\hline & UNC1079 & $>100(3)$ & $>100(3)$ \\
\hline \multirow{3}{*}{ 6His-D274A } & DMSO & $>100(3)$ & $>100(3)$ \\
\hline & UNC1215 & No binding (3) & No binding (3) \\
\hline & UNC1079 & $>100(3)$ & $>100(3)$ \\
\hline 6 His-CBX7 & DMSO & No binding (6) & No binding (6) \\
\hline
\end{tabular}

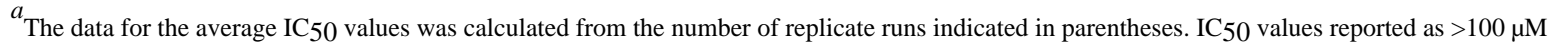
showed binding that was too weak to quantify based on the top concentration tested, whereas no binding is reported for cases where no signal was observed. 